\title{
Simulationsbasierte Entwicklung energieeffizienter Steuerungsstrategien für Materialflusssysteme
}

\author{
Prof. Dr.-Ing. Thorsten Schmidt, Dr.-Ing. Frank Schulze \\ Technische Universität Dresden \\ Lehrstuhl für Technische Logistik
}

\begin{abstract}
Die Kosten für die Energieversorgung eines Materialflusssystems übersteigen die Investitionskosten oft schon nach wenigen Jahren. Mit steigenden Energiepreisen wird dieser Zeitraum kürzer. Bei der Bewertung der Lebenszykluskosten eines Materialflusssystems rücken deshalb die Energiekosten immer mehr in den Vordergrund. Viele Möglichkeiten zur Energieeffizienz-Steigerung sind zwar bereits bekannt, jedoch wird davon meist nur punktuell, nicht aber systematisch Gebrauch gemacht. Dies gilt insbesondere für die Ebene der Anlagenbzw. Systemsteuerung.
\end{abstract}

Die Bemühungen um eine Steigerung der Energieeffizienz in der Intralogistik fokussieren bislang auf die Optimierung fördertechnischer Komponenten (Leichtbau, Antriebe). Damit wird jedoch nur das Symptom behandelt, nicht aber die Ursache: Der energetische Aufwand für Transportvorgänge - das Symptom - muss zweifellos reduziert werden. Das geschieht auch bereits. Zugleich ist aber sicherzustellen, dass zur Lösung einer konkreten logistischen Aufgabenstellung - der Ursache Transporte nur im tatsächlich erforderlichen Umfang und in der richtigen Weise durchgeführt werden. Dafür Sorge zu tragen, ist Aufgabe einer energieeffizienten Anlagensteuerung.

Die Forschungsarbeiten an der TU Dresden verfolgen drei Ziele: (a) Der Zusammenhang zwischen der mechanischen Leistung und dem elektrischen Energieverbrauch typischer Fördertechnik-Komponenten soll allgemeingültig beschrieben werden. (b) Es sollen Routinen entwickelt werden, mit denen in der ereignisdiskreten Simulation die mechanische Leistung einer Anlage und der resultierende elektrische Energieverbrauch bestimmt wird. (c) Mithilfe der Simulation soll gezeigt werden, wie und in welchem Umfang optimierte Steuerungsstrategien die Energieeffizienz von Materialflusssystemen steigern.

\section{Status Quo}

Vanderlande Industries B.V. schlägt bei der „Energiekostenaufteilung eines großen Logistikdienstleisters" $24 \%$ der Intralogistik zu, wovon immerhin $48 \%$ der „Fördertechnik-, Lager- \& Kommissioniertechnik“ zuzuschreiben sind [GuT09a]. So ist es nicht verwunderlich, dass beim 18. Deutschen Materialfluss-Kongress 2009 in München „in den zahlreichen Fachvorträgen insbesondere die Themen Energieeffizienz und Green Logistics im Fokus“ standen [Irr09]. Schon auf der CeMAT 2008 wurde ein Energierückspeise-Modul der PSB Intralogistics GmbH mit 
dem MM-Logistik-Award ausgezeichnet [Unr09]. Diese wenigen Beispiele belegen deutlich, dass das Thema Energieeffizienz sehr weit oben auf der Agenda der Intralogistik-Branche steht.

Von Systemtechnikanbietern werden bereits in der Angebotsphase genaue, z.T. pönalisierte Angaben zum Energieverbrauch ihrer Anlagen verlangt, die sie heute aber aufgrund der Dynamik und Stochastik des Systembetriebs nicht machen können. Es ist zwar bekannt, dass der mittlere Energieverbrauch ein gewisser Prozentsatz der installierten Leistung ist - belastbare Schätzungen zum Verbrauch bei bestimmten Lastszenarien sowie zum Verlauf und zur Höhe von Lastspitzen gibt es aber praktisch nicht.

Durch eine intelligente Steuerung sind in Fördertechnikanlagen Energieeinsparungen bis zu 30\% ... 50\% (It. [Vlz07] insb. im Teillastbetrieb) möglich. Bei der Entwicklung einer energieeffizienten Anlagensteuerung tritt so neben die traditionellen Optimierungsziele (maximaler Durchsatz, minimale Durchlaufzeit, minimale Investitionskosten) ein weiteres Optimierungsziel: minimale Betriebskosten.

Die Entwicklung intelligenter, energieeffizienter Steuerungen benötigt geeignete Modelle zur Abschätzung des Energieverbrauchs. Diese sind heute aber nicht verfügbar. Es fehlen Werkzeuge, um die Einsparpotentiale einzelner Maßnahmen exakt zu beziffern und systematisch Strategien zu ihrer Erschließung zu entwickeln. Dies gilt insbesondere für die Ebene der Anlagen- bzw. Systemsteuerung.

[Die08] schreiben: „Zusammenfassend lässt sich sagen, dass die energieeffiziente Produktion voraussetzt, dass effiziente Prozesse auf effizienten Maschinen umgesetzt werden müssen und die Maschinen zusätzlich effizient betrieben und gesteuert werden müssen." Es gelingt ihnen, den Energieverbrauch bestimmter Produktionsmaschinen mithilfe eines Modells bemerkenswert genau vorherzusagen und sie kommen zu dem Fazit: „Aufgrund seiner Struktur eignet es [das Modell] sich sehr gut als Grundlage zur Optimierung von Maschinen sowie zur Optimierung der Steuerung und Nutzung." Kurz: Ohne Modell keine Optimierung. Es ist Anliegen der Autoren, entsprechende Modelle für die Fördertechnik zu schaffen.

\subsection{Effizienzreserven}

Unruh [Unr09] formuliert: „Und weil nicht gemachte Wege nun einmal die energiesparendsten sind, werden Softwarelösungen künftig mehr und mehr zum entscheidenden Faktor eines kostenbewussten Betriebs fördertechnischer Anlagen.“ Auch Hahn-Woernle [HWo09] fragt zu recht: „Warum muss eine Förderanlage permanent und auf Höchstleistung laufen, auch wenn gegen Nachmittag die Auftragseinlastung abnimmt? Eine leistungsstarke Lagersoftware mit intelligentem Kennzahlensystem kann das individuell steuern." Vergleichbares schlagen z.B. auch Mouzon et al. [MYT07, S. 4251 ff.] vor.

Es ist natürlich naheliegend, eine Maschine dann auszuschalten, wenn der Energieaufwand für Abschaltung und Wiederanlauf geringer ist als der (c) 2011 Logistics Journal : Proceedings - ISSN 2192-9084 
Energieverbrauch im Standby-Modus während des überbrückten Zeitraums. Und selbst damit sind noch nicht einmal alle Einsparpotentiale erschlossen, denn auch bei Volllast gibt es dringende und weniger dringende Transportaufträge. Bislang wird aber nur in Ausnahmefällen (z.B. bei Gepäckförderanlagen in der Flughafentechnik) die verbleibende Transportzeitreserve eines Auftrags zum Entscheidungskriterium für seinen Transportweg und seine Transportgeschwindigkeit gemacht. Ähnlich argumentieren Mouzon et al. [MYT07, S. 4253 ff.]. Sie empfehlen für nicht kritisch ausgelastete Ressourcen in einem Fertigungssystem eine abwechselnde BatchBearbeitung und Abschaltung und wägen so zwischen Reduzierung des Energieverbrauchs und einer Erhöhung der Durchlaufzeit ab.

In einer Firmenschrift von Daifuku [Dai09] heißt es: „Es besteht die grundsätzliche Möglichkeit, die Gassen zunächst nur mit einem Regalbediengerät zu betreiben. Zu einem späteren Zeitpunkt [...] können die Gassen mit weiteren Geräten nachgerüstet werden. Diese Flexibilität bleibt auch nach der Nachrüstung erhalten: So kann in Schwachlastphasen - je nach Auslastungsgrad - eines der beiden AKL-Geräte innerhalb einer Gasse automatisch in seiner Dynamik reduziert oder sogar stillgelegt werden." An gleicher Stelle findet sich eine weitere interessante Abwägung: Die Vorzone von Hochregallagern kann statt traditioneller Stetigfördertechnik durch sog. „Sorting Transfer Vehicle - kurz STV“ in „STV-Loops“ angebunden werden, denn „die spätere Ergänzung weiterer Fahrzeuge zur Leistungserhöhung ist deutlich einfacher und kostengünstiger zu realisieren als mit konventioneller Fördertechnik. “1

Fraglos ist, dass eine allein von wirtschaftlichen Erwägungen getriebene Optimierung des Fahrzeugeinsatzes zumeist auch positive Energieeffizienz-(Neben-)Effekte hat. Und selbst dort sind auch heute noch erhebliche Optimierungspotentiale zu erschließen. So schätzt die PSI Logistics $\mathrm{GmbH}$ ein, dass durch ein intelligentes Staplerleitsystem „bis zu $30 \%$ der Fahrzeugbewegungen vermieden werden [können]“ und die Locanis AG spricht von „75\% aller Fahrten ohne Ware auf der [Stapler-] Gabel“ [GuT09b]. Dennoch darf nicht übersehen werden: Bislang war die Minimierung der Investitionskosten (d.h. eine möglichst geringe Fahrzeuganzahl) das dominierende Optimierungskriterium. Es ist aber grundsätzlich denkbar, dass eine stärkere Gewichtung der Betriebskosten (welche mit steigenden Energiepreisen künftig an Bedeutung gewinnen) zu anderen Lösungen führt - bei denen z.B. eine größere Fahrzeuganzahl kürzere Leerfahrten bedingt. So meinen auch Bozer \& Yen [BoY96]: „Existing dispatching rules [...] may lead to sizable empty travel (also known in industry as ,dead-heading'), which reduces system efficiency."

\footnotetext{
${ }^{1}$ Das ist grundsätzlich richtig, unterschlägt aber zum einen, dass Unstetigförderer ein (ggf. sehr ungünstiges) Totzeitverhalten haben (welches Stetigförderer nicht kennen), und darüber hinaus, dass sich bei ungünstigen Last- und Layout-Verhältnissen ein (unproduktiver) Leerfahrtanteil von über $50 \%$ einstellen kann. Dennoch illustriert auch dieses Beispiel, wie eng die elektrische Leistungsaufnahme und die „logistische Leistungsabgabe“ eines fördertechnischen Systems miteinander verknüpft sind und lässt erahnen, welche Bedeutung dabei auch den Steuerungs- und Optimierungsstrategien zukommt.
} 
In eine andere Richtung gehen die Betrachtungen zur Energieeffizienz in der Recyclingindustrie [Mey09]. Sie heben wiederholt das Spitzenlastmanagement hervor. Anhand mehrerer Beispiele (dito, S. 70, 97 f., 102) zeigen sie, dass Investitionen in zusätzliche Soft- und Hardware zum Lastmanagement sich bereits innerhalb von ca. 2 Jahren amortisieren. Das rechtfertigt die Annahme, dass auch in automatisierten fördertechnischen Systemen unter vergleichsweise übersichtlichen (d.h. kontrollierbaren) Rahmenbedingungen z.B. durch die zeitliche Entzerrung von Transportoperationen mit vergleichsweise geringem Aufwand ähnlich hohe Einsparungen $z u$ erzielen sind. Das legen auch an verschiedenen Stellen veröffentlichte Zahlen zum Einsparungspotential von Zwischenkreiskopplung und Netzrückspeisung nahe - selbst wenn sie insgesamt kein konsistentes Bild ergeben:

\begin{tabular}{lrl}
\hline \multicolumn{1}{c}{ Anwendungsfall } & Einsparung & Quelle \\
\hline RBG (Zwischenkreiskopplung) & $50 \ldots 60 \%$ & {$[$ Scm10] } \\
Paletten-RBG (Netzrückspeisung) & bis $59 \%$ & {$[$ Grü10] } \\
Servoantrieb (Netzrückspeisung) & $52 \%$ & {$[$ Hkl08] } \\
Hubantrieb (Netzrückspeisung) & $32 \ldots 46 \%$ & {$[$ Vot09] } \\
RBG (Netzrückspeisung) & $10 \ldots 25 \%$ & [Dai09] \\
AKL-RBG (Netzrückspeisung) & bis 20\% & [Grü10] \\
RBG (Zwischenkreiskopplung) & bis 15\% & [Grü10] \\
Bahnbetrieb (Netzrückspeisung) & $8 \%$ & [Scm09] \\
\hline
\end{tabular}

Tabelle 1: Energieeinsparungen in verschiedenen Anwendungsfällen

\subsection{Modellierungsansätze}

Einen methodisch interessanten Zugang beschreiben Dietmair et al. [Die08] mit sog. „zustandsbasierten Energieverbrauchsprofilen“ von Werkzeugmaschinen. Sie schreiben: „Ausgehend von der Beobachtung, dass das Leistungsaufnahmeverhalten einer Maschine primär davon abhängt, welchen Betriebszustand sie einnimmt, wurde die [...] Modellierung in Form eines Zustandsgraphen gewählt." Es gelingt ihnen damit, den Energieverbrauch bei Prozessen der spanenden Metallbearbeitung auf ca. $5 \%$ genau vorherzusagen. Zwar ist fraglich, ob ihr Ansatz in jedem Fall auf die Fördertechnik zu übertragen ist, ${ }^{2}$ er hat aber aus Sicht der Simulation einigen Charme, weil Betriebszustände in der Simulation leicht zu erfassen sind. Aus genau diesem Grunde bietet die WirthLogistik GmbH seit einiger Zeit mit dem Produkt „EcoSim“ eine entsprechende Softwarelösung an. Hier kann für einzelne Elemente im Simulationsmodell die Leistungsaufnahme angegeben werden. Diese ist bei Eco-Sim jedoch nur abhängig vom Betriebszustand (z.B. für ein RBG das Anfahren, Heben und Halten, vgl. [WLG09]), nicht aber von Transportgut oder Bewegungsablauf.

\footnotetext{
${ }^{2}$ Einerseits macht es einen Unterschied, ob ein Stapel Leerpaletten oder eine beladene Palette mit $1.000 \mathrm{~kg}$ Gewicht transportiert wird - andererseits wird dieser Unterschied bei einem $30 \mathrm{~m}$ hohen Regalbediengerät mit einem Eigengewicht von 20 t eher unerheblich sein.
} 
Somit können aus den Modellen nur relativ grobe Aussagen zum effektiven Energieverbrauch abgeleitet werden.

Dass grundsätzlich nur selten einfache (z.B. lineare) Abhängigkeiten zu erwarten sind, bestätigen auch Verl et al. [VHD09]. Sie stellen für Werkzeugmaschinen fest: „In keinem Fall kann von der Anschlussleistung direkt auf den realen Verbrauch geschlossen werden, ebenso wenig vom reinen Prozesswirkungsgrad auf die Gesamteffizienz." Betrachtet man gelegentlich veröffentlichte Messdaten zur Leistungsaufnahme fördertechnischer Komponenten (wie z.B. in der folgenden Abbildung), erscheint diese Einschätzung plausibel. Aus diesem Grunde gehen auch Draganescu et al. [DGD03] einen anderen Weg: Sie untersuchen (ebenso wie Dietmair et al. [Die08]) Prozesse der spanenden Metallbearbeitung und betrachten die Abhängigkeit des Wirkungsgrades einer Maschine von den Prozessparametern als „Response Surface“. Diese beschreiben sie näherungsweise durch ein Polynom der Logarithmen der Prozessparameter.

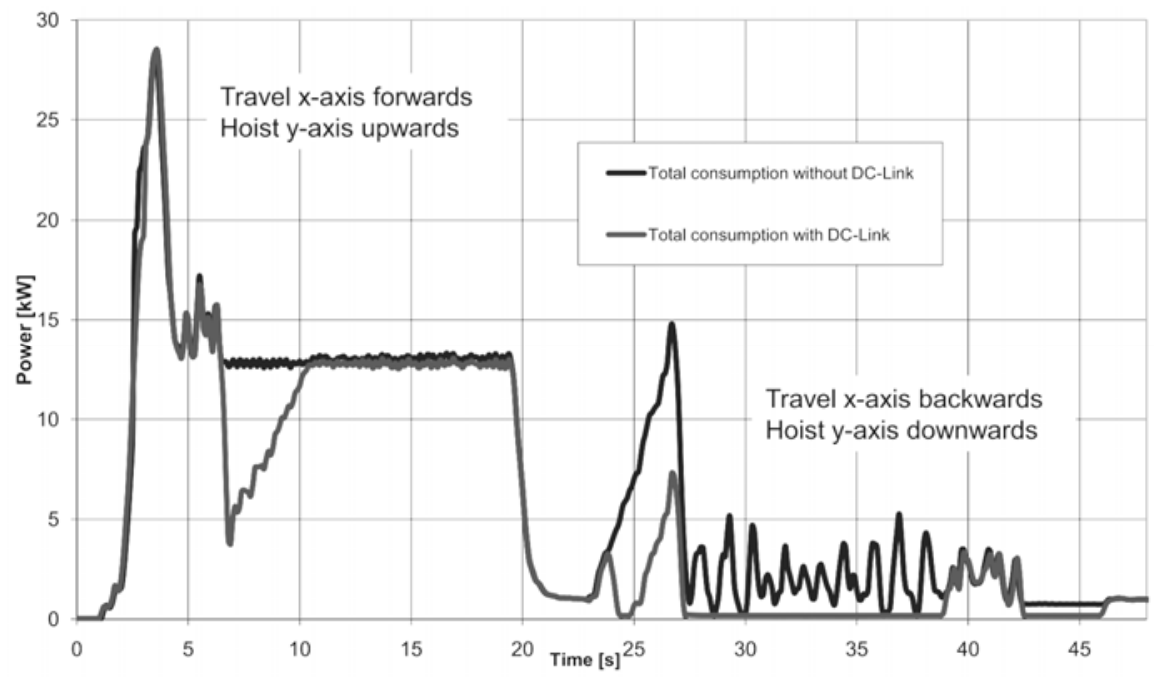

Abbildung 1: Messung der elektrischen Leistungsaufnahme eines RBG ohne (dunkler) bzw. mit (heller) Zwischenkreiskopplung [Scm10]

\section{Lösungsansatz}

Der Entwurf und die Erprobung von Steuerungsstrategien für Logistik- und Produktionssysteme geschehen heute überwiegend mithilfe der ereignisdiskreten Simulation. Im Bereich der Anlagensteuerung hat sich das Simulationssystem AutoMod als Quasi-Standard etabliert. Bislang wird es jedoch (nur) genutzt, um logistische Kenngrößen (z.B. die Durchlaufzeit) zu optimieren, nicht aber den Energieverbrauch der Anlagen. Wie letzterer optimiert werden könnte, zeigen folgende Beispiele:

- Eine Effizienzsteigerung kann durch die Zusammenfassung von Transporten (Sammeltransporte, Batchbildung) erreicht werden. Ein Wandel der Steuerungsphilosophie von „Förderer wartet auf Gut“ zu „Gut wartet auf Förderer“ kann helfen, Energieeinsparpotentiale ohne Abstriche an der Qualität der 
logistischen Leistung zu erschließen (z.B. zeitunkritische Transporte, Ausschöpfen von Transportzeitreserven).

- Im Niedriglastfall kann die Transportgeschwindigkeit anlagenweit auf einen verbrauchsoptimalen Wert reduziert werden [HWo09] und es können Fahrzeuge bzw. Förderabschnitte außer Betrieb genommen werden.

- Moderne, hocheffiziente Elektromotoren haben prinzipbedingt eine höhere Massenträgheit, was beim Anfahren nachteilig ist. Der Vorteil ihres höheren Wirkungsgrades kommt daher nur im Dauerbetrieb mit konstanter Drehzahl zum Tragen. Dem muss die Anlagensteuerung Rechnung tragen [SLS09].

Im Rahmen eigener Untersuchungen haben die Autoren die Möglichkeiten einer simulativen Bestimmung der Leistungsaufnahme fördertechnischer Systeme bewertet. Dabei wurde im Simulationssystem AutoMod das unten abgebildete Modell implementiert. Es handelt sich um zwei unabhängige Verteilwagen, welche jeweils drei Förderstrecken miteinander verbinden, auf denen Paletten von vorn/rechts nach hinten/links transportiert werden.

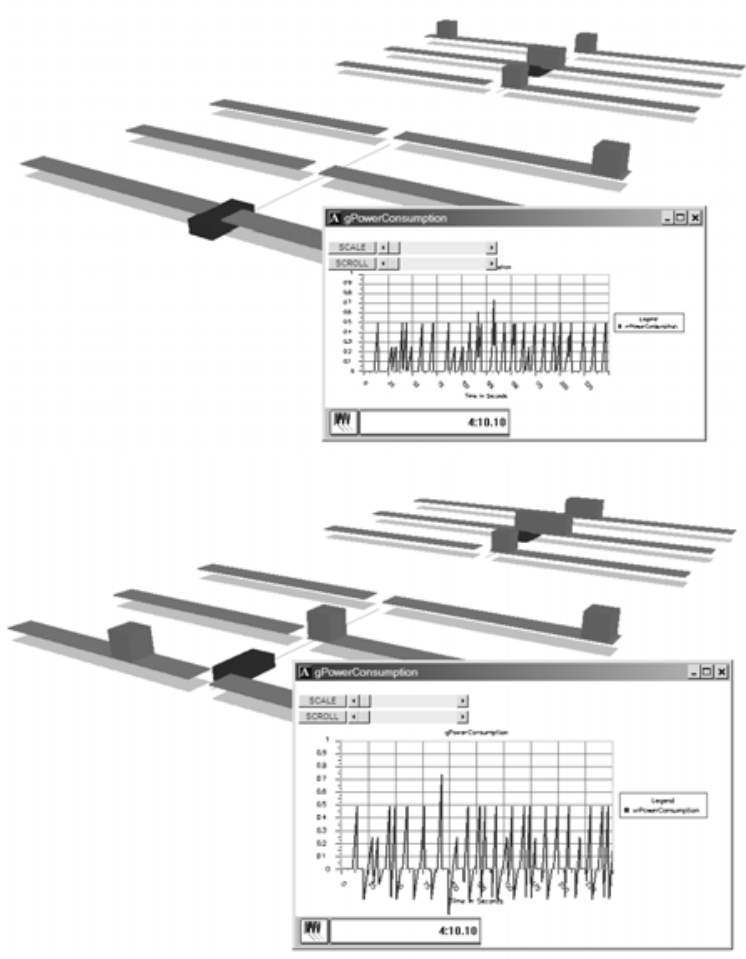

Abbildung 2: Simulation der mechanischen Leistungsaufnahme von zwei Verteilwagen ohne (links) bzw. mit (rechts) „Rückspeisung“ (die Diagramme stellen den Energieverbrauch über der Zeit dar)

Es zeigte sich, dass mit AutoMod die mechanische Leistungsaufnahme fördertechnischer Prozesse vergleichsweise einfach ermittelt werden kann. Dazu wird für Transporteinheiten und Transportmittel die Masse parametriert. Für alle Bewegungen im Modell können Wege, Zeiten, Geschwindigkeiten, Beschleunigungen und Verzögerungen während des Modelllaufs ermittelt werden. 
Damit lässt sich im beschriebenen Fall z.B. auch das Einsparungspotential für eine Zwischenkreiskopplung der beiden Verteilwagen bestimmen: Im linken Beispiel operieren beide Verteilwagen unabhängig voneinander; im rechten Beispiel hingegen prüft jeder Verteilwagen unmittelbar vor dem Anfahren, ob der jeweils andere Verteilwagen in Bewegung ist. Ist das der Fall, wird der Anfahrvorgang so lange zurückgestellt, bis der bewegte Verteilwagen zu bremsen beginnt. Dessen Bremsenergie wird dann für die Beschleunigung genutzt.

Es konnte nachgewiesen werden, dass durch „Rückspeisung" eine Energieeinsparung von ca. $35 \%$ (insgesamt) zu erzielen ist. Sie wird (wegen des Zurückstellens von Anfahrvorgängen) mit einer Leistungseinbuße (Durchsatz) von ca. $10 \%$ erkauft. Die effektive Energieeinsparung beträgt demnach ca. $30 \%$ (je Transport). Dabei wird hier der Begriff „Rückspeisung“ bewusst apostrophiert, denn im Simulationsmodell wird mechanische Energie zwischen den Verteilwagen ausgetauscht - während im technischen System elektrische Energie rückgespeist wird, was zwangsläufig mit Wirkungsgradverlusten verbunden ist. Nichts desto trotz erlaubt das beschriebene Modell eine Abschätzung des abzuschöpfenden Einsparungspotentials (obere Grenze) sowie eine Bewertung des Einflusses der Dispositions- und Steuerungsstrategien auf die mechanische (resp. elektrische) Leistungsaufnahme einerseits und die logistische Leistung andererseits.

Die folgende Abbildung zeigt ein weiteres Modell, mit dem die Energieaufnahme eines Stetigförderers untersucht wurde. Im Modell werden Behälter von vorn über eine Schleife zu einer „Bearbeitungsstation“ transportiert. Im linken Teil der Abbildung erfolgt die Bearbeitung in einem zufälligen, exponentialverteilten Zeitraum, im rechten Teil der Abbildung erfolgt sie getaktet. Dadurch wird ein Rückstau mit Stop-and-Go-Betrieb vermieden, welcher bei der wiederholten Beschleunigung ganzer Behältergruppen zu den im linken Diagramm erkennbaren Lastspitzen führt. Allein durch die Vertaktung erreicht die maximale Lastaufnahme so nur ca. $80 \%$ der Spitzenwerte des ungetakteten Falls.
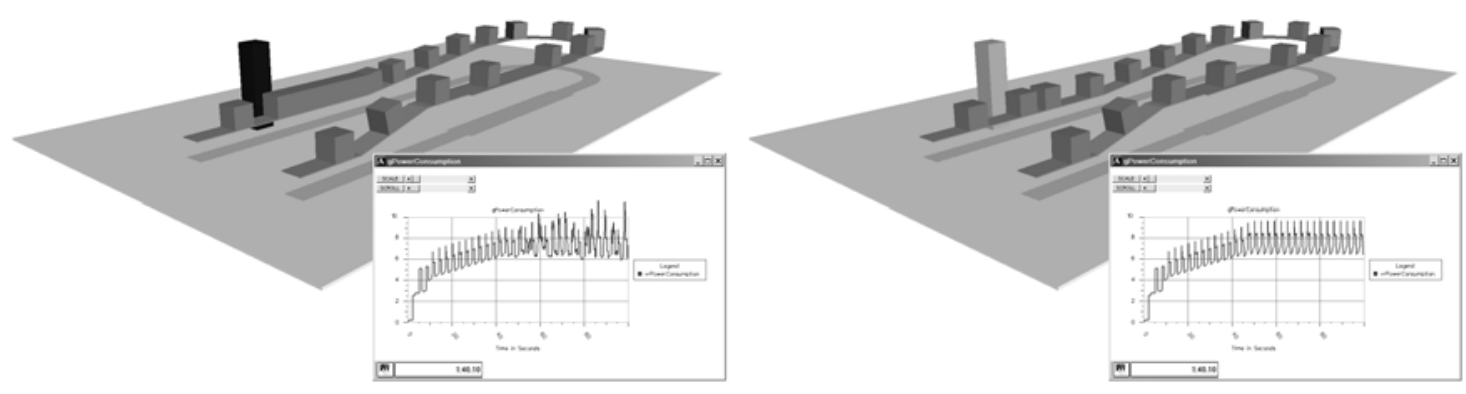

Abbildung 3: Simulation der mechanischen Leistungsaufnahme eines Stetigförderers mit zufälliger (links) bzw. getakteter (rechts) „Bearbeitung“ der Transporteinheiten

Auch dieses Beispiel illustriert auf einfache Weise den Einfluss von Betriebsstrategien auf den Energieverbrauch der Fördertechnik. Es zeigt zugleich aber recht deutlich die Lücke auf, welche durch künftige Forschungsarbeiten erst zu schließen ist: In beiden vorgestellten Modellen wird nur die in sehr kurzen (c) 2011 Logistics Journal : Proceedings - ISSN 2192-9084 
Abtastintervallen verrichtete mechanische Arbeit und damit die aufgenommene mechanische Leistung, mithin eine „abstrakte“ Größe, bestimmt - welche elektrische Leistungsaufnahme dem gegenübersteht, ist jedoch nicht bekannt. Wie dieser Lückenschluss erfolgen kann, zeigen die folgenden „Thesen“:

1. Die primäre Ursache für den Energieverbrauch in fördertechnischen Systemen ist die Verrichtung mechanischer Arbeit, insbesondere beim Heben und Beschleunigen.

2. Die Umwandlung von elektrischer Energie in mechanische Arbeit erfolgt in fördertechnischen Komponenten über eine große Zahl von Schritten (Umrichter, Elektromotor, Getriebe, Kraftübertragung), deren Einzelwirkungsgrade nichtlinear und betriebszustandsabhängig sind. Daher ist eine theoretische Berechnung des Gesamtwirkungsgrades nur sehr ungenau möglich.

3. Das effektive Wirkungsgrad-Kennfeld einzelner fördertechnischer Komponenten kann besser nach der Messung des Energieverbrauchs bei der Ausführung von Referenzspielen erfasst und mithilfe von Näherungsformeln beschrieben werden.

4. Die verrichtete mechanische Arbeit beim Transport einzelner Fördereinheiten kann mit hoher Genauigkeit in der Simulation ermittelt werden.

5. Da im Simulationsmodell bekannt ist, welche fördertechnische Komponente für einen konkreten Transportvorgang verantwortlich ist, kann mithilfe ihres Wirkungsgrad-Kennfeldes berechnet werden, welche elektrische Leistungsaufnahme dieser Transportvorgang erfordert.

Damit ist der Weg für künftige Arbeiten sehr genau vorgezeichnet.

\section{$3 \quad$ Fazit}

Bei Recherchen zum Stand der Technik fiel auf, dass bislang vergleichsweise wenige relevante Arbeiten zu Fragen der Energieeffizienz in der Intralogistik in rein wissenschaftlichen Publikationen dokumentiert wurden. Der überwiegende Teil der Veröffentlichungen hat einen engen Industriebezug, zumeist dokumentieren Hersteller einzelne besonders energieeffiziente Produkte. Was jedoch fehlt, ist der Blick auf das logistische Gesamtsystem. Diesen Eindruck bestätigen Sbihi \& Eglese [ShE10]. Sie schreiben: "There is not much literature that links VRSP [Vehicle Routing and Scheduling Problem] models with the Green Logistics issues [...]. Most articles are concerned with objectives of minimizing economic costs."

Das hat verschiedene Gründe: Zum einen hat wohl überhaupt erst die Ölpreisentwicklung (vgl. untenstehendes Bild) im letzten Jahrzehnt das Thema der Energieeffizienz nachdrücklich ins allgemeine Bewusstsein gerückt. Hinzu kommt, dass entsprechende Lösungsansätze in der Logistik nicht allein mit den Methoden der Operationsforschung entwickelt werden können, denn es werden (Berechnungs-) Modelle benötigt, mit denen einem konkreten Transportvorgang ein konkreter Energieverbrauch (in Form von elektrischer Energie oder auch bspw. als 
Verbrennungskraftstoff) zugeschrieben werden kann. Diese Modelle stehen heute aber noch nicht zur Verfügung. Sie zu schaffen, ist Zielstellung für künftige Arbeiten an der Professur für Technische Logistik der Technischen Universität Dresden. Diese Arbeit können nur die Ingenieurwissenschaften (und hier insb. der Maschinenbau und die Elektrotechnik) leisten und genau das ist auch die Motivation für die Autoren.

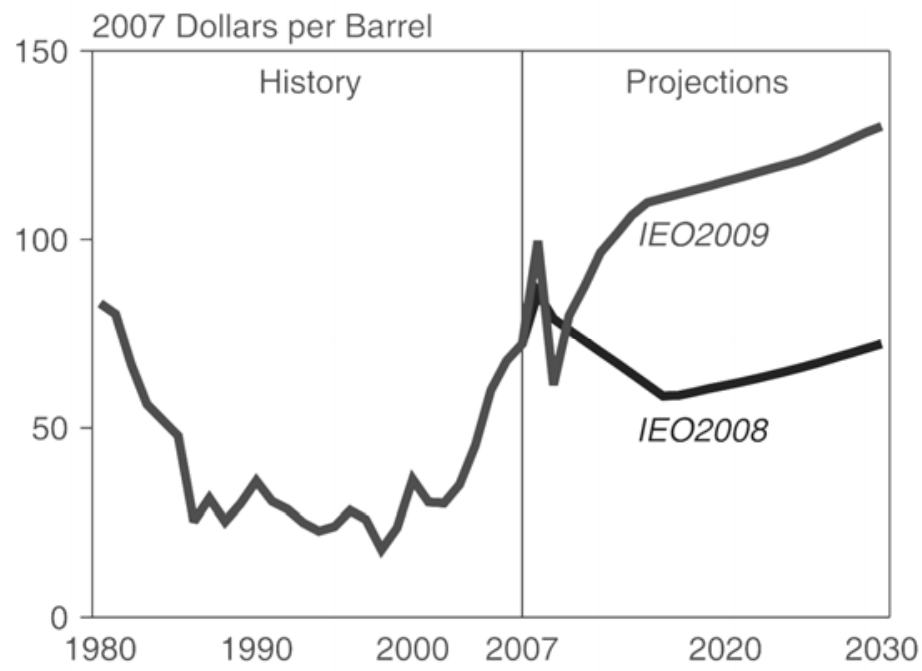

Abbildung 4: Historische Entwicklung und Prognose des Ölpreises [EIA09]

So kommen auch Sbihi \& Eglese [ShE10] zu dem Schluss: „It is expected that as environmental factors assume increasing importance, the effective use of combinatorial optimization theories and techniques will be needed to meet the challenges of new problems."

\section{Literatur}

[BoY96] Bozer, Y. A.; Yen, C.: Intelligent dispatching rules for trip-based material handling systems. In: Journal of Manufacturing Systems, Bd. 15 (1996), Nr. 4, S. 226-239. - DOI: 10.1016/0278-6125(96)84549-3

[Dai09] Daifuku Europe Ltd., Hrsg.: Energieeffizienz im Focus der Intralogistik. In: MyLogistics.net (29. Mai 2009). - URL: www.mylogistics.net/de/news/ themen/key/news1046822/jsp

[Die08] Dietmair, A.; Verl, A.; Wosnik, M.: Zustandsbasierte Energieverbrauchsprofile : Eine Methode zur effizienten Erfassung des Energieverbrauchs von Produktionsmaschinen. In: wt Werkstattstechnik online, $\mathrm{Bd} .98$ (2008), Nr. 7/8, S. 640-645

[DGD03] Draganescu, F.; Gheorghe, M.; Doicin, C. V.: Models of machine tool efficiency and specific consumed energy. In: Journal of Materials Processing Technology, Bd. 141 (1. Oktober 2003), Nr. 1, S. 9-15

[EIA09] Energy Information Administration: International Energy Outlook 2009. Mai 2009. - verfügbar unter www.eia.doe.gov/oiaf/ieo

[Grü10] Grün, O.: Steigerung der Energieeffizienz in der Intralogistik : Energieverbrauch und Energierückgewinnung. In: 6. Symposium Logistik 
Innovativ (Prien am Chiemsee, 4. Mai 2010)

[GuT09a] Günthner, W. A.; Tenerowicz, P.: Wege zu einer energieeffizienten Intralogistik. In: Brauwelt (2009), Nr. 27, S. 773-775

[GuT09b] Günthner, W. A.; Tenerowicz, P.: Change to Green : Wege zu einer energieeffizienten Logistik. Vortrag, 1. Symposium Energieeffizienz und Klimawandel - C.L.I.M.A.T.E. 2009, München, 5. Mai

[HWo09] Hahn-Woernle, C.: Ohne intelligentes Warehouse Management System keine energieeffiziente Logistik. In: MM Logistik online (11. Sep. 2009)

[Hkl08] Henkel, R.: Energieeffizienz in der Automatisierung. In: automotion: Das $B \& R$ Kundenmagazin, Bd. 8 (2008), Nr. 12, S. 8-11. - verfügbar unter: www.automotion. info/uploads/media/MM-E00840.699.pdf

[Irr09] Irrgang, R.; Seemann, A.; von Janczewski, B.: Lösungen für eine nachhaltige Logistik. In: Logistik für Unternehmen, Bd. 23 (2009), Nr. 4/5, S. 46-50

[Mey09] Meyer, J.; Schubert, A.; Trautmann, A.; Kausch, C.; Kirschbaum, S.; Probst, T.; Killmann, D.; Julius, J.: Leitfaden Energieeffizienz für die Recyclingindustrie. März 2009. - URL: www.nrw-spart-energie.de/ _database/_data/datainfopool/Leitfaden_Recycling_DINA4.pdf

[MYT07] Mouzon, G.; Yildirim, M. B.; Twomey, J.: Operational methods for minimization of energy consumption of manufacturing equipment. In: International Journal of Production Research, Bd. 45 (15. September 2007), Nr. 18-19, S. 4247-4271. - DOI: 10.1080/00207540701450013

[ShE10] Sbihi, A.; Eglese, R. W.: Combinatorial optimization and Green Logistics. In: Annals of Operations Research, Bd. 175 (März 2010), Nr. 1, S. 159175. - DOI: 10.1007/s10479-009-0651-z

[SLS09] Schmidt, T.; Leonhardt, T.; Schulze, F.: Logistiktechnologie - Bausteine exzellenter Wertschöpfung. In: Erfolg kommt von innen : Kongressband : 26. Deutscher Logistik-Kongress (Berlin, 21. Oktober 2009), hrsg. v. T. Wimmer \& H. Wöhner, S. 602-634. - ISBN: 978-3-87154-401-9

[Scm10] Schumacher, M.: Energiesparen mit System. Vortrag, Automatisierungstage 2010, Emden: Fachhochschule, 3. Februar. - verfügbar unter www.automatisierungstage.de

[Scm09] Schumacher, O., Hrsg.: Nachhaltigkeitsbericht 2009 : Deutsche Bahn AG. Juni 2009. - verfügbar unter www.deutschebahn.com

[Unr09] Unruh, V.: Der nicht gemachte Weg ist stets der effizienteste. In: MM MaschinenMarkt online (10. November 2009). - URL: www.maschinenmarkt.vogel.de /index.cfm?pk=238126

[VHD09] Verl, A.; Huf, A.; Dietmair, A.: Wie Automatisierung Energie spart: Direkte und indirekte Maßnahmen in der Gerätetechnik. In: Energy 2.0 (März 2009), S. 26-31. - verfügbar unter www.energy20.net mit more@clickCode E2039052

[Vot09] Vogt, J. P.: Antriebssysteme mit dem Drive Solution Designer energieeffizient auslegen. 2009. - Firmenschrift der Lenze Drive Systems $\mathrm{GmbH}$, Hameln 
[Vlz07] Volz, G.: Informationsblätter Fördertechnik. hrsg. v. Deutsche EnergieAgentur $\mathrm{GmbH}$ (dena). - verfügbar unter www.industrieenergieeffizienz.de

[WLG09] WirthLogistik GmbH, Hrsg.: Übersicht WirthSim Elemente : Version 1.35. Dezember 2009. - Firmenschrift, verfügbar unter www.wirthsim.com 